\title{
Persistent behavioural blindness after early visual deprivation and active visual rehabilitation: a case report
}

\author{
SYNNÖVE CARLSON, LEA HYVÄRINEN, AND ANTTI RANINEN \\ From the Department of Physiology, University of Helsinki
}

SUMMARY Early long lasting binocular deprivation results in behavioural blindness in both man and experimental animals. However, few reported cases show that visual rehabilitation may improve visual behaviour. A 34-year-old man who had experienced 30 years of binocular deprivation due to bilateral cataracts received visual rehabilitation for one year. The rehabilitation included training in eye-hand co-ordination, recognition of objects, evaluation of distance and size, and mobility training. Despite signs of recovery of visual functions the patient never started to use vision in his normal life. The negative outcome of the rehabilitation is partly attributed to the patient's motivational problems and to the relatively short rehabilitation time. Visual rehabilitation may be successful when started immediately after the corrective operation on the eyes when the level of motivation is also high.

Early binocular visual deprivation has a profound effect on the visual behaviour of monkeys and humans after the visual deprivation has ended. ${ }^{12}$ There are only a few case reports on the recovery from long lasting early blindness. ${ }^{2-6}$ The process of potential recovery is always long and difficult. ${ }^{27}$ Visual rehabilitation was attempted in only two case reports, and it gave encouraging results. ${ }^{27}$ Electrophysiological studies on visually deprived monkeys have shown that early binocular visual deprivation results in behavioural blindness, though the eyes, the optic nerves, the lateral geniculate nucleus, and the primary visual cortex function well. ${ }^{89}$ Although there are changes in the function of the primary visual cortex, ${ }^{8}$ they do not explain the poor visual behaviour of such animals. It has been shown that early binocular visual deprivation affects the processing of visual signals in the more central parts of the cortex, ' resulting in difficulty in interpreting visual information.

In our first report of the effects of visual rehabilitation on a patient recovering from long lasting early blindness we described the slow but continuous recovery of the visual functions. ${ }^{2}$ Apkarian ${ }^{6}$ de-

Correspondence to Synnöve Carlson, MD, Department of Physiology, University of Helsinki, Siltavuorenpenger $20 \mathrm{~J}, 00170$ Helsinki, Finland. scribed the effect of visual training with the aid of the Tactile Vision Substitution system on a girl who had been deprived of form vision from the age of 3 months to 11 years. Despite many difficulties during the training period there was clear evidence of improved visual abilities. These encouraging results induced us to attempt again to enhance the recovery of visual functions of a patient by means of active visual rehabilitation.

\section{Case report}

The patient was a 34-year-old man from Zambia. No documents about his early visual status or visual development were available. According to his own report he had become blind at the age of 4 years. The cause of his blindness was unknown, but he had developed bilateral cataracts. He had some visual memories, but it remained uncertain whether his visual status before the blindness had been normal or abnormal. At the age of 16 a bilateral cataract removal was performed and he was given spectacles after the operation. However, they, broke after two months, whereafter he remained without spectacles. He thought that the operation had been a failure and had continued living as a blind person. Despite his handicap he became an educated man in Zambia. He 
learned Braille and studied in a school for the blind. He was found to be a talented student and was given the opportunity to come to Finland to be trained as a teacher for the visually handicapped. We thought that he might have a chance to learn to use his residual vision if he would get proper visual rehabilitation. His motivation to learn to use his sight was diminished because 18 years had elapsed from the time of his cataract operation. Physiologically the time lag meant that the duration of visual deprivation had continued after the corrective operation.

VISUAL STATUS

The patient's visual status before rehabilitation was as follows. The eyes were aphakic. There were no scars from superficial infection and no corneal disease or iritis. Intraocular tensions were normal. The media were clear and the fundi normal except for an inverted disc in the left eye. Refraction in both eyes was $+11 \cdot 0$. With aphakic correction vision in the right eye was light perception and some perception of hand movements. In the left eye the vision was perception of the direction of an $8 \mathrm{~mm}$ black line at a distance of $3 \mathrm{~cm}$. With respect to colours he could recognise red, blue, yellow, green, and black. As aphakic correction the patient was prescribed spectacles of $+13 \cdot 0$.

The visually evoked potential (VEP) was recorded twice during the rehabilitation period. The stimulus was a black-and-white $21.5^{\circ} \times 16.4^{\circ}$ square-wave checkerboard pattern. The mean luminance of the checkerboard pattern was $5.5 \mathrm{~cd} . \mathrm{m}^{-2}$ with a contrast of $95 \%$ between the black and white squares. The viewing distance was $100 \mathrm{~cm}$. The checks were reversing at $2 \mathrm{~Hz}$, and analysis was triggered by every second pattern reversal. The electrodes were situated at 01 and 02 and referred to linked ears. In each experimental session we recorded the VEP twice for two different check sizes $\left(7^{\circ}\right.$ and $\left.50^{\prime}\right)$. With large checks there was a response through the left eye. There was no response through either eye when small checks were used. The latency for P100 was $148 \mathrm{~ms}$ in the first recording session, which took place five months after rehabilitation was initiated. The second VEP was done three months after the first; the latency for P100 had shortened to $120 \mathrm{~ms}$. In the second recording we tested the VEP also to diffuse light flickering once a second. The stimulator was a Grass PS-22 Photostimulator with a mean luminance of $197 \mathrm{~cd} \cdot \mathrm{m}^{-2}$. The response was normal except for a lengthened latency for P100 (120 ms).

\section{VISUAL BEHAVIOUR BEFORE REHABILITATION}

The patient moved about as a blind person using a long cane and tactile exploration. He kept his eyes almost closed and did not pay any visual attention to the surroundings. He read only in Braille. When talking to a person he did not try to look at him. When sitting at a table he made no effort to find things on the table by sight but rather used his hands for exploration. It made no difference to him whether the room was lit or not.

\section{REHABILITATION PROGRAMME}

The rehabilitation followed the outlines of the programme described in detail previously. ${ }^{2}$ The rehabilitation took place once a week in the Department of Physiology in Helsinki or outdoors in parks and streets. The sessions lasted 1-2 hours. In addition to the authors two other persons contributed to the rehabilitation-Dr Ninel Nezlina (USSR) who was working with $\mathrm{SC}$ at the time and Mr Ilkka Linnankoski.

The procedure at the indoor sessions was as follows. The patient was first taught to direct his gaze towards an object and to follow its movement with his eyes. He was taught eye-hand co-ordination using finger paints which enabled him to see the place on paper he had touched with his finger. Later eye-hand co-ordination was sharpened by painting, picking up small things, catching balloons, throwing balls, and by grasping objects near and far. His ability to perceive two and three dimensional forms was developed by showing him pictures of squares, triangles, and circles and by giving him cubes and balls. He was taught to combine visual and tactile information by letting him always touch the objects carefully which he could not initially recognise visually. Later, simple forms were replaced by familiar objects of everyday life (kitchen utensils, pens, books, fruits, etc.). He was taught to evaluate whether an object was near or far, big or small, high or low, thin or thick.

Part of the session was used for mobility training. When indoors he was taught to move about in a room, to recognise open and closed doors, furniture, and windows. When outdoors he was taught to estimate distances, to keep his eyes open continuously, and to pay attention to his feet while walking. During the sessions the patient often told us that he became tired of using his sight and continued to cooperate for two hours at the most. He often wanted to have a short break during which he closed his eyes for a couple of minutes.

The same set of tests which we used to detect possible progress in the first patient's visual abilities ${ }^{2}$ were used also with this patient. The tests were: (1) The labyrinth. The patient had to draw a line between two curved lines. (2) A line between two spots. The patient was asked to draw a line from one spot to another. (3) The colouring test. The patient was asked to fill with colour the forms of a triangle, circle, and a square drawn on paper. (4) The picking 
test. The patient was asked to pick up 20 pellets from a white board as quickly as possible. (5) The post box test. The patient was asked to put eight blocks of four different forms into a box through a cover which had a hole for each form. The tests were performed once a month.

\section{Results}

At the beginning the patient was enthusiastic about the rehabilitation, but when he realised that recovery was questionable he became less eager to try to practice. However, he came to the sessions and, despite the difficulties, made some progress.

\section{SIGNS OF PROGRESS}

Two weeks after having received the spectacles the patient spontaneously stated that he could no longer stay in a dark room and wanted to turn on the lights to be more aware of the surroundings visually. After 16 weeks from the beginning of the rehabilitation he said that he had for the first time recognised paintings on the walls and had enjoyed looking at them. Later he was given some simple pictures to look at and was able to describe roughly the contents of the pictures. In one picture there was a horse on the lawn. The patient said there was an animal on it. However, he missed all details and had only a faint idea of the contents of the picture. He had learned to recognise kitchen utensils and fruits by sight. For instance, he could distinguish between an orange, a lemon, and a
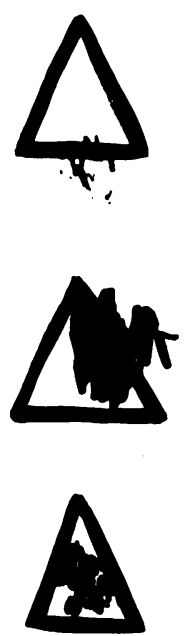

Fig. 1 The patient's progress in colouring the forms of a triangle, circle and a square is shown. Top forms were coloured after one month, middle forms after four months of rehabilitation, and bottom forms at the end of the rehabilitation.
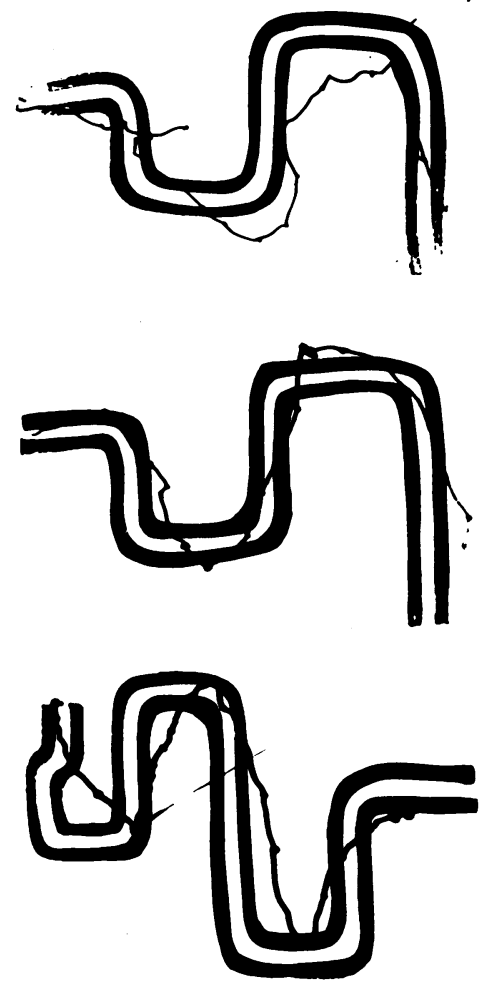

Fig. 2 The results of the labyrinth test. Top figure shows the performance after one month, middle figure after three months of rehabilitation, and the bottom figure at the end of the rehabilitation.

grapefruit and tell by visual inspection whether there was a plum, tomato, or an egg on the table. $\mathrm{He}$ learned to recognise practically all the vegetables and fruits available in Finland.

RESULTS OF THE TESTS

Colouring test. The patient learned to avoid the contours of the figures but failed to fill the total area of the picture with colour. His progress in colouring the forms can be seen in Fig. 1 .

The labyrinth. The patient never learned to master the labyrinth well and was unwilling to perform the task. His progress in this test is shown in Fig. 2.

Post box. The patient gave up trying to perform this test. It was necessary to combine visual and tactile information to master the test. He had to look at the holes on the cover of the box and was allowed to find the right block by exploring the blocks available.

Picking test. The patient's performance in this test is shown graphically in Fig. 3. He was able to grasp 


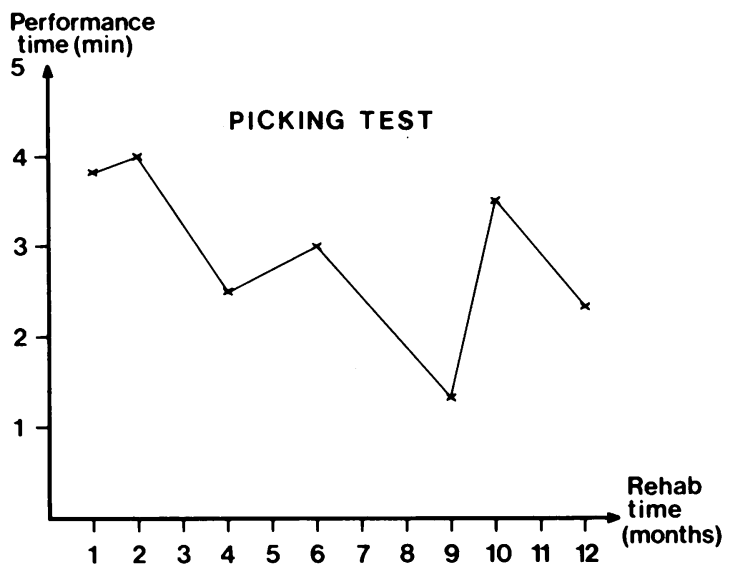

Fig. 3 The patient's performance in the picking test is shown graphically as a function of the rehabilitation time.

the pellets with almost complete accuracy and could also see the more distant pellets without great effort. However, his performance varied.

A line between two spots. The patient found it difficult to place his hand accurately on the starting spot. He learned to draw the line fairly well between the two spots, as can be seen in Fig. 4.

When asked to use vision only, the patient was able to avoid bumping into obstacles, and he could find and recognise on the table such objects as books,
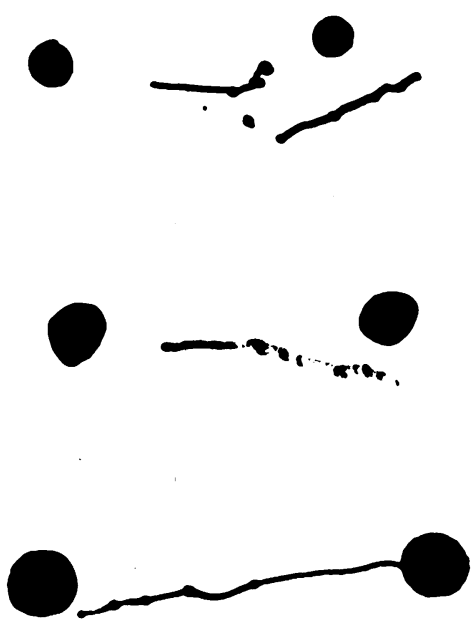

Fig. 4 Illustration of the patient's ability to draw a line between two spots. Top example was done after two months, middle example after seven months of rehabilitation, and the bottom one at the end of the rehabilitation. glasses, and pencils. He could tell how many jars, for instance, there were on the table. But he never used these abilities in his normal life. He could tell just by looking around how many people there were in the room, but he never learned to recognise a person by his looks. When outdoors the patient did not rely on his vision at all and moved about using his cane and preferably the guidance of another person. Sometimes the patient made remarks about the surroundings which indicated that he had visual abilities which were not in active use. Once when we went to the market place he spontaneously asked why there were so many tents there. The tents were the shelters of the salespersons. However, even such incidents did not encourage the patient to continue to use his sight actively; he continued living as a blind person. Thus the final outcome of the rehabilitation, which lasted one year, was negative for the patient.

\section{Discussion}

The idea of enhancing the recovery of functions after sensory deprivation by active rehabilitation originated from the results of studies of the effect of visual deprivation on the function of different areas of the central nervous system.

It has been shown that binocular deprivation has a profound effect on the function of the posterior parietal cortex of monkeys ${ }^{10}$ and on the superior colliculus of cats ${ }^{11}$ and rats ${ }^{12}$. The type of functional changes in the cells in these areas is quite similar. The representation of vision decreases and the representation of others modes of perception increases. It seems that during the early sensitive period of life there is competition between the modalities in relation to their influence on the function of the cells in the central nervous system. In the case of binocular visual deprivation, the representation of vision in these areas decreases.

In our first case report of visual rehabilitation ${ }^{2}$ the patient showed slow but continuous improvement of her visual functions. The rehabilitation was successful. Contrary to this first experience, the visual behaviour of our second patient did not improve significantly during rehabilitation lasting one year.

The reasons for the negative result are numerous. Our patient came from a culture where emphasis is placed on basic health problems and where it is usually very difficult for a handicapped person to gain a respected position in the society. Since our patient had achieved a fine status as a blind man, he was worried from the beginning about the changes that a partially improved vision might bring about when he returned to his country. This attitude severely affected his motivation to practise and to overcome difficulties during rehabilitation. The long interval between the 
corrective operation on his eyes and the beginning of the rehabilitation also made him less motivated.

The visual acuity was checked again in the middle of the rehabilitation period and was found to be unchanged. Previously it has been reported that binocular deprivation may arrest the development of visual acuity but does not disrupt the acuity level already established. ${ }^{13}$

On two successive VEP measurements with an interval of three months the latency for P100 shortened from $148 \mathrm{~ms}$ to $120 \mathrm{~ms}$ with a patterned stimulus. However, on the basis of this study alone it is not possible to determine the value of VEP as a measure of recovery of visual function. The fact that VEP could be elicited with flash stimuli proves that the primary visual cortex was functioning. It has been suggested that VEP offers a means of testing the residual function of the visual cortex of behaviourally blind patients. ${ }^{14}$ Our findings support this idea.

From study of the first patient it was concluded that the improvement in the visual functions was directly related to the amount of practise. It was only after one and a half years that the first patient was able to improve her visual abilities by herself. Accordingly, one year of visual rehabilitation may not have been long enough to encourage the second patient to use his vision continuously to get improved visual functions. During rehabilitation the patient's performance improved in many visual tasks, but his visual functions never developed enough to be useful for him in everyday life.

After early binocular deprivation visual rehabilitation may enhance the recovery of visual functions. However, the longer the deprivation period lasts, the more important it is that the rehabilitation is supportive and continues long enough to assure good results.
We thank Mr Ilkka Linnankoski and Dr Ninel Nezlina for participating in the rehabilitation, and Mrs Katriina Lauren and Mr Raimo Lauren for preparing the pictures. The work was supported by a grant from the Sigrid Juselius Foundation.

\section{References}

1 Hyvärinen J, Hyvärinen L, Färkkilä M, Carlson S, Leinonen L. Modification of visual functions of the parietal lobe at early age in the monkey. Med Biol 1978; 56: 103-9.

2 Carlson S, Hyvärinen L. Visual rehabilitation after long lasting early blindness. Acta Ophthalmol (Kbh) 1983; 61: 701-13.

3 Valvo A. Sight restoration after long-term blindness: the problems and behaviour patterns of visual rehabilitation. New York: American Foundation for the Blind, 1971.

4 Ackroyd C, Humphrey NK, Warrington EK. Lasting effects of early blindness. A case study. J Exp Psychol 1974; 26: 114-24.

5 Gregory RL, Wallace JG. Recovery from early blindness: a case study. Experimental Psychology Society Monograph No. 2. Cambridge: Heffer, 1963.

6 Apkarian PA. Visual training after long term deprivation. A case report. Int J Neurosci 1983; 19: 65-84.

7 von Senden M. Raum- und Gestaltauffassung bei operierten Blindgeborenen vor und nach der Operation. Leiptzig: Barth, 1932.

8 Wiesel TN, Hubel DH. Comparison of the effects of unilateral and bilateral eye closure on cortical unit responses in kittens. $J$ Neurophysiol 1965; 28: 1029-40.

9 Hubel DH. The visual cortex of normal and deprived monkeys. Sci Am 1979; 67: 532-43.

10 Hyvärinen J, Hyvärinen L, Carlson S. Effect of binocular deprivation on parietal association cortex in young monkeys. Doc Ophthalmol Proc Ser 1981; 30: 177-85.

11 Rauschecker JP, Harris LR. Auditory compensation of the effects of visual deprivation in the cat's superior colliculus. Exp Brain Res 1983; 50: 69-83.

12 Vidyasagar TR. Possible plasticity in the rat superior colliculus. Nature 1978; 275: 140-1.

13 Regal DM, Boothe R, Teller DY, Sackett GP. Visual acuity and visual responsiveness in dark reared monkeys (Macaca nemestrina). Vision Res 1976; 16: 523-30.

14 Regan D, Regal DM, Tibbles JAR. Evoked potentials during recovery from blindness recorded serially from an infant and his normally sighted twin. Electroencephalogr Clin Neurophysiol 1982; 54: 456-68.

Accepted for publication 5 December 1985. 